\title{
Searches for Leptonic B decays at BABAR
}

\author{
Guglielmo De Nardo, representing the BABAR collaboration \\ Physics Department, University of Napoli, \\ Compl. Univ. Monte Sant'Angelo, via Cintia \\ 80126 Napoli, Italy
}

\begin{abstract}
We present the most recent results on the leptonic B decays $B \rightarrow l v, B \rightarrow l v \gamma$, based on the data collected by the BABAR experiment [1] at PEP-II, an asymmetric $e^{+} e^{-}$collider at the center of mass energy of the $\Upsilon(4 S)$ resonance.
\end{abstract}

Keywords: B meson, B meson Leptonic Decays

PACS: $13.20 .-\mathrm{v}, 13.25 . \mathrm{Hw}$

\section{THEORETICAL CONTEXT}

In the Standard Model (SM), the purely leptonic decays $B \rightarrow l v$ proceeds via quark annihilation into a virtual $\mathrm{W}$ boson. The SM branching fraction is cleanly calculated as:

$$
\mathscr{B}(B \rightarrow l v)=\frac{G_{F}^{2} m_{B} m_{l}^{2}}{8 \pi}\left[1-\frac{m_{l}^{2}}{m_{B}^{2}}\right]^{2} \tau_{B} f_{B}^{2}\left|V_{u b}\right|^{2}
$$

In the context of the SM, the measurement of the branching fractions of the purely leptonic B decays permits a theoretically clean extraction of the B decay constant $f_{B}$, using $\left|V_{u b}\right|$ measurement from semileptonic $b \rightarrow u$ decays. However, leptonic decays are also sensitive to physics beyond the SM. For instance, in two-Higgs doublet models[2] and in MSSM[3], it can be mediated also by charged Higgs bosons:

$$
\begin{aligned}
\mathscr{B}(B \rightarrow l v)_{2 H D M} & =\mathscr{B}(B \rightarrow l v)_{S M} \times\left(1-\tan ^{2} \beta \frac{m_{B}^{2}}{m_{H}^{2}}\right)^{2} \\
\mathscr{B}(B \rightarrow l v)_{S U S Y} & =\mathscr{B}(B \rightarrow l v)_{S M} \times\left(1-\frac{\tan ^{2} \beta}{1+\varepsilon_{0} \tan \beta} \frac{m_{B}^{2}}{m_{H}^{2}}\right)^{2},
\end{aligned}
$$

where $m_{H}$ is the mass of the charged Higgs, $\tan \beta$ is the ratio of the vacuum expectation values of the Higgs fields, and $\varepsilon_{0}$ parametrizes higher orders SUSY contributions. The branching fraction measurement, therefore, can constrain the parameter space of extensions of the SM. The measurement of the branching ratios of different lepton flavors $R_{B}^{\mu / \tau}=\mathscr{B}(B \rightarrow \mu v) / \mathscr{B}(B \rightarrow \tau v)$ and $R_{B}^{e / \tau}=\mathscr{B}(B \rightarrow e v) / \mathscr{B}(B \rightarrow \tau v)$ can also be used as a probe of lepton flavor violation [4].

The radiative leptonic decay $B \rightarrow l v \gamma$ proceeds via quark annihilation into a virtual $\mathrm{W}$ boson after radiating a photon, which removes the helicity suppression but introduces an additional suppression due to the electromagnetic coupling constant. The differential branching fraction as a function of photon energy involves a vector and an axial form 
factors, $f_{V}$ and $f_{A}$ :

$$
\frac{d \mathscr{B}}{d E_{\gamma}}\left(B \rightarrow l v_{l} \gamma\right)=\frac{\alpha_{e m} G_{F}^{2}\left|V_{u b}\right|^{2}}{48 \pi^{2}} m_{B}^{4} \tau_{B}\left[f_{V}^{2}\left(E_{\gamma}\right)+f_{A}^{2}\left(E_{\gamma}\right)\right](1-y) y^{3},
$$

where $y=2 E_{\gamma} / m_{B}$. Most models [5] suggest $f_{A}=f_{V}$, others [6] suggest $f_{A}=0$ The total branching fraction is given in Ref. [7] as:

$$
\mathscr{B}\left(B \rightarrow l v_{l} \gamma\right)=\frac{\alpha_{e m} G_{F}^{2}\left|V_{u b}\right|^{2}}{288 \pi^{2}} f_{B}^{2} m_{B}^{5} \tau_{B}\left(Q_{u} / \lambda_{B}^{2}-Q_{b} / m_{B}\right),
$$

where $Q_{u, b}$ are the u- and b-quark charges, $m_{b}$ is the b-quark mass, and $\lambda_{B}$ is the first moment of the B meson distribution function, expected to be of he order of $\Lambda_{Q C D}$, but estimated theoretically with a large uncertainty.

\section{EXPERIMENTAL RESULTS}

\section{Untagged $B \rightarrow e v$ and $B \rightarrow \mu v$}

This analysis [8] is performed in a sample of 468 millions of $B \bar{B}$ pairs. The two body decay kinematics of the $B \rightarrow l v$, where 1 is an electon or a muon, provides a strong signature for this decay. The charged lepton momentum is indeed expected to be monochromatic in the B rest frame with $p \sim m_{B} / 2$, and it can be accurately reconstructed. We select the lepton candidate requiring its momentum in the center of mass frame to be $2.4 \mathrm{GeV} / \mathrm{c}<p_{C M}<3.2 \mathrm{GeV} / \mathrm{c}$ and applying loose particle identification criteria. All the reconstructed charged tracks and neutral energy deposits in the calorimeter are combined to measure the momentum and energy of the companion B. We use an extended maximum likelihood fit to extract signal and background yields using as probability density functions the output of a Fisher discriminant based on five observables and the energy-substituted mass of the companion B meson $m_{E S}=\sqrt{E_{\text {beam }}^{2}-p_{B}^{2}}$. We do not find a statistically significant signal and set a $90 \%$ C.L. upper limit on the branching fraction, using a Bayesian approach. The results are reported in table 1.

TABLE 1. Signal yield, efficiency and 90\% C.L. upper limits for the $B \rightarrow e v, \mu v$ branching fractions

\begin{tabular}{lrrr}
\hline & $\begin{array}{r}\text { Fitted Signal } \\
\text { events }\end{array}$ & Efficiency & $\begin{array}{r}\mathbf{9 0 \%} \text { CL } \\
\text { Upper Limit }\end{array}$ \\
\hline$B \rightarrow e v$ & $18 \pm 14$ & $(4.7 \pm 0.3) \%$ & $1.9 \times 10^{-6}$ \\
$B \rightarrow \mu \nu$ & $1 \pm 15$ & $(6.1 \pm 0.2) \%$ & $1.0 \times 10^{-6}$ \\
\hline
\end{tabular}

\section{$B \rightarrow l v$ with semileptonic tags}

This analysis [9] is performed in a sample of 459 millions of $B \bar{B}$ pairs. We first reconstruct the semileptonic decays of one of the two B produced at the $\Upsilon(4 S)$ (tag 
B), then we search for evidence of the $B \rightarrow l v$ decay in the rest of the event.

The $\tau$ decay is detected using particle identification criteria and $\pi^{0}$ reconstruction in the following $\tau$ decay modes: $\tau \rightarrow e v v, \tau \rightarrow \mu \nu v, \tau \rightarrow \pi v$, and $\tau \rightarrow \rho v$. We use topological and kinematics variables to suppress the background, and define a discriminating variable $E_{\text {extra }}$ summing the energy of all the calorimeter clusters not unassigned either to the tag B or the the signal B. Since no extra energy besides beam backgrounds or from recostruction inefficiency is expected in signal events, $E_{\text {extra }}$ is very effective in separating the signal from the background. The result, shown in Table 2, excludes the hypothesis of a null signal at $2.4 \sigma$ level.

Combining this result with the hadronic tagged analysis [10] $B(B \rightarrow \tau v)=(1.8 \pm$ $0.9 \pm 0.4) \times 10^{-4}$ we obtain $B(B \rightarrow \tau v)=(1.8 \pm 0.6) \times 10^{-4}$.

We search for $B \rightarrow e v$ and $B \rightarrow \mu v$ requiring the signal candidate charged track momentum $p>2.52(2.45) \mathrm{GeV} / \mathrm{c}$ for electrons (muons). We do not find evidence of signal in the electron and muon channels and set $90 \%$ C.L. upper limits to the branching fractions, using a frequentistic approach. The results are reported in Table 2.

TABLE 2. Signal yield, efficiency and branching fractions (90\% C.L when upper limits) for purely leptonic B decays

\begin{tabular}{lrrrr}
\hline & $\begin{array}{r}\text { Expected background } \\
\text { events }\end{array}$ & $\begin{array}{r}\text { Observed } \\
\text { events }\end{array}$ & Efficiency & Branching fraction \\
\hline$B \rightarrow e v$ & $24 \pm 11$ & 17 & $(36.9 \pm 1.5) \times 10^{-4}$ & $<7.7 \times 10^{-6}$ \\
$B \rightarrow \mu \nu$ & $15 \pm 10$ & 11 & $(27.1 \pm 1.2) \times 10^{-4}$ & $<11.0 \times 10^{-6}$ \\
$B \rightarrow \tau \nu$ & $521 \pm 31$ & 610 & $(10.54 \pm 0.41) \times 10^{-4}$ & $1.8 \pm 0.8 \pm 0.1 \times 10^{-4}$ \\
\hline
\end{tabular}

\section{$B \rightarrow l v \gamma$ with hadronic tags}

This analysis [11] is performed on a sample of 465 millions of of $B \bar{B}$ pairs. We reconstruct the tag $\mathrm{B}$ in its most abundand charmed hadronic decays, then we search for evidence of a $B \rightarrow e v \gamma$ or a $B \rightarrow \mu v \gamma$ decay in the rest of the event. We use a likelihood ratio based on 5 observables to reduce combinatorial background, search for one opposite charged lepton passing particle identification requirements and apply $\pi, \omega$ and $\eta$ vetos. The signal photon candidate is the most energetic neutral cluster in the event, and the missing momentum, required to be in the detector acceptance, is used to estimate the momentum of the undetected neutrino.

We do not find a significant signal in either the electron or muon modes, therefore we set $90 \%$ C.L. upper limits on the branching fractions. Since the no kinematical requirement is imposed on signal observables, the results, reported in table 3 , are model independent.

We select in a more stringent but model dependent way the signal, requiring a cut on the angle between the lepton and the photon, and on the angle between the photon and the neutrino. We consider two possible models for the form factors, one with $f_{A}=f_{V}$ and a second with the $f_{A}=0$. The selection requirements, optimized for the best upper limit, in both cases reduce the efficiency by $\sim 40 \%$. The (model dependent) results are reported in Table 4 
TABLE 3. Signal yield, efficiency and 90\% C.L. model independent upper limits for the B radiative leptonic decays

\begin{tabular}{lrrrr}
\hline & $\begin{array}{r}\text { Expected background } \\
\text { events }\end{array}$ & $\begin{array}{r}\text { Observed } \\
\text { events }\end{array}$ & Efficiency & Branching fraction \\
\hline$B \rightarrow e v \gamma$ & $2.7 \pm 0.1 \pm 0.4$ & 4 & $(7.8 \pm 0.1 \pm 0.3) \times 10^{-4}$ & $<17 \times 10^{-6}$ \\
$B \rightarrow \mu v \gamma$ & $3.4 \pm 0.7 \pm 0.7$ & 7 & $(8.1 \pm 0.1 \pm 0.3) \times 10^{-4}$ & $<26 \times 10^{-6}$ \\
combined & & & & $<16 \times 10^{-6}$ \\
\hline
\end{tabular}

TABLE 4. Signal yield, efficiency and $90 \%$ C.L. upper limits for B radiative leptonic decays with assumptions on form factors

\begin{tabular}{lrrr}
\hline & Expected events & Observed events & 90\% CL Upper Limit \\
\hline$B \rightarrow e v \gamma\left(f_{A}=f_{V}\right)$ & $0.6 \pm 0.1$ & 0 & $8.4 \times 10^{-6}$ \\
$B \rightarrow \mu v \gamma\left(f_{A}=f_{V}\right)$ & $1.0 \pm 0.4$ & 0 & $6.7 \times 10^{-6}$ \\
combined & & & $3.0 \times 10^{-6}$ \\
\hline$B \rightarrow e v \gamma\left(f_{A}=0\right)$ & $1.2 \pm 0.4$ & 3 & $29 \times 10^{-6}$ \\
$B \rightarrow \mu v \gamma\left(f_{A}=0\right)$ & $1.5 \pm 0.5$ & 2 & $22 \times 10^{-6}$ \\
combined & & & $18 \times 10^{-6}$ \\
\hline
\end{tabular}

\section{CONCLUSIONS}

Leptonic B decays are an excellent probe for new Physics, branching fraction measurements being complementary to the direct searches at high energy machines. Current experimental measurements are in agreement with Standard Model expectations, but, being already capable to constrain parameters of New Physics models today, they will be golden channels for the foreseen super flavor factories.

\section{REFERENCES}

1. BABAR collaboration, B. Aubert et al., Nucl. Instrum. Meth. A 4791 (2002)

2. W. Hou, Phys. Rev. D. 482342 (1993).

3. A. G. Akeroyd and S. Recksiegel J. Phys. G: Nucl. Part. Phys. 292311 (2003).

4. G. Isidori and P. Paradisi, Phys. Lett. B 639499 (2006).

5. E. Lunghi, D. Pirjol and D. Wyler, Nucl. Phys. B 649337 (2003).

6. G. Burdman, T. Goldman, and D. Wyler, Phys. Rev. D 51111 (1995).

7. G. P: Korchemsky, D. Pirjol and T. M. Yan, Phys. Rev. D 61, 114510 (2000).

8. BABAR collaboration, B. Aubert et al., Phys. Rev. D 79, 091101(R) (2009).

9. BABAR collaboration, B. Aubert et al., arXiv:0809.4027 [hep-ex].

10. BABAR collaboration, B. Aubert et al., Phys. Rev. D 77, 011107(R) (2008).

11. BABAR collaboration, B Aubert et al., arXiv:0907.1681 [hep-ex]. 